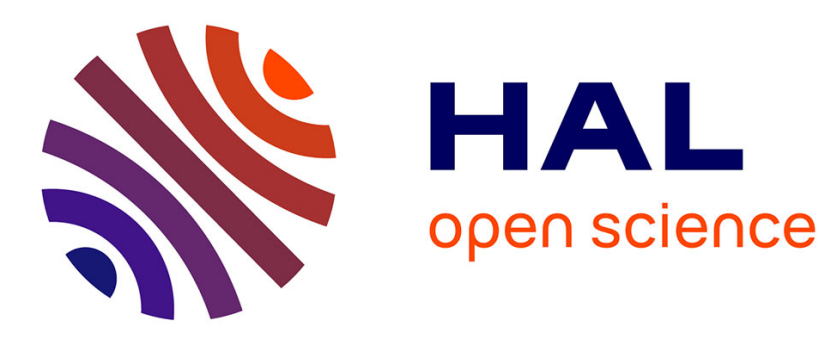

\title{
Reasoning with spatial relations over high-content images
}

Nicolas Lomenie

\section{To cite this version:}

Nicolas Lomenie. Reasoning with spatial relations over high-content images. IEEE World Congress on Computational Intelligence, WCCI'2010, hosting the International Joint Conference on Neural Networks, IJCNN'2010, Jul 2010, barcelone, Spain. hal-00497810

\section{HAL Id: hal-00497810 https://hal.science/hal-00497810}

Submitted on 6 Jul 2010

HAL is a multi-disciplinary open access archive for the deposit and dissemination of scientific research documents, whether they are published or not. The documents may come from teaching and research institutions in France or abroad, or from public or private research centers.
L'archive ouverte pluridisciplinaire HAL, est destinée au dépôt et à la diffusion de documents scientifiques de niveau recherche, publiés ou non, émanant des établissements d'enseignement et de recherche français ou étrangers, des laboratoires publics ou privés. 


\title{
Reasoning with spatial relations over high-content images
}

\author{
Nicolas Loménie
}

\begin{abstract}
Spatial relation and configuration modeling issues are gaining momentum in image analysis and pattern recognition fields in the perspective of mining high-content images or large scale image databases in a more expressive way than purely statistically. Continuing our previous efforts whereby we developed specific efficient morphological tools performing on mesh representation like Delaunay triangulations, we propose to formalize spatial relation modeling techniques dedicated to unorganized point sets. We provide an original mesh lattice framework more convenient for structural representations of large amount of image data by the means of interest points sets and their morphological analysis. The set of designed numerical operators is based on a specific dilation operator making it possible the representation of concepts like "between" or "left of" over sparse representations such as graphs. Then, for the sake of illustration and discussion, we apply these new tools to high-level queries in microscopic histo-pathological images and structural analysis of macroscopic images.
\end{abstract}

\section{INTRODUCTION}

Visual cognition or cognitive vision is a long-term goal aiming at putting together computer vision analysis tools and artificial intelligence reasoning methodologies in order to design robust systems for smart vision-based interfaces. As a first step towards this ambitious integrated research purpose, image understanding modules providing high-level image descriptions is a cornerstone, at least for the final user to seamlessly interact with visual data.

The major issue can be referred as the semantic gap filling that is the coincidence between low-level features given by the signal and the high-level concepts used to describe and handle images at a semantic level. Few works [1], [2] have dealt with such an issue in a sound theoretical way. For instance, in the field of spatial relation modeling, [7], [4] made a great extent of work for radiometric image modeling defined over regular grids, that is the mainstream way in which visual data are handled. In this work, we made an attempt to extend these seminal ideas to visual data represented over sparse representations such as interest point sets. In particular, Delaunay triangulation representations are favored for the neighborhood embedding of these visual data defined over irregular grids (see Fig. 1).

These mathematically well defined tools make it possible to consider enhanced visual systems with enabled reasoning capabilities. Not only they provide relevant algorithms to explore large images for instance in a more formal way as a robot would be expected to do by anchoring spatial relation concepts into the signal representation either as an image or a point set visual data. In addition, they open new opportunities

Nicolas Loménie is with the Department of Computer Sciences, University of Paris Descartes and with CNRS - IPAL, I2R, National University of Singapore; email: nicolas.lomenie@mi.parisdescartes.fr

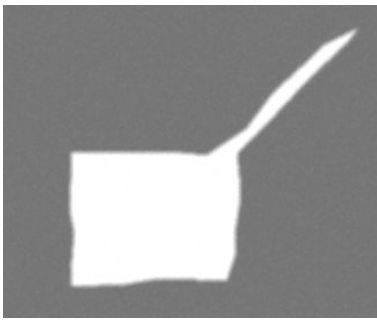

(a)

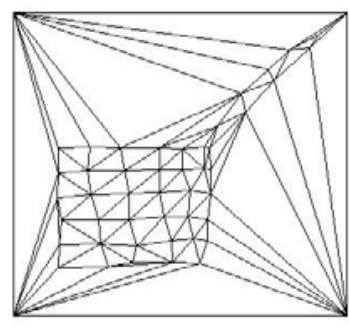

(b)
Fig. 1. Different representations of the same object: (a) gray intensity image $I$ and (b) geometric mesh $M$

to perform high-level queries over large image databases featuring specific spatial organization requirements.

Last, coming with the challenging great amount of visual data to process due to dramatic progress in both resolution and speed of the acquisition devices, satellite image as well as medical image end-users urge for more interactive, qualitative ways of interaction with the visual data they use to assess or diagnose daily critical situations. Even at the level of more long-term biological research, smart interactions with visual data such as microscopic information turn to become practical requirements due to the high-content partly related to the size - of these images (see below Fig. 2 and Table I for an illustration of the amount of data for a single patient biopsy in histopathological imaging). Of course, satellite imaging with new resolution up to $0.7 \mathrm{~cm}$ a pixel over 24000x24000 pixel size images will have to face the same kind of issues in the near future.

TABLE I

HISTOPATHOLOGICAL IMAGE DATA

\begin{tabular}{|c|c|c|}
\hline & Size (pixels) & Size (bytes) \\
\hline x1 & $1018 \times 768$ & 3.05 Mo \\
\hline x10 & $3664 \times 2763$ & 39.54 Mo \\
\hline x20 & $14657 \times 11054$ & 632.48 Mo \\
\hline x40 & $58630 \times 44216$ & 7.77 Go \\
\hline
\end{tabular}

As image size scales up exponentially according to the progress in the field of optics, interactive time applications can not use mainstream image analysis algorithms for computational complexity issues. Working on sparse representations such as interest point sets can be a solution to this kind of real time issues by focusing on informative parts on the image. For instance, working on points of interest in the image of Fig. 2(a) scales down the size of visual data from 7.77 Go up to 1.5 Go. In that perspective, the adaptation of existing techniques to point set representations and so on to graph related representation should be a prospective field of research [5], [10]. Section II presents the theoretical 
The rank filtering operators need the computation of two additional values $e_{T}$ and $d_{T}$ for each triangle $T$, defined by:

$$
\begin{aligned}
& e_{T}=\min \left\{\phi_{T^{\prime}} \mid T^{\prime} \in \nu(T)\right\} \\
& d_{T}=\max \left\{\phi_{T^{\prime}} \mid T^{\prime} \in \nu(T)\right\}
\end{aligned}
$$

The neighborhood $\nu(T)$, playing the key role of a structuring entity, is the set of all triangles $T$ of $\operatorname{del}(S)$ sharing at least one vertex with the triangle $T$, that is:

$$
\nu(T)=\left\{T^{\prime} \in \operatorname{Del} \mid T^{\prime} \cap T \neq \emptyset\right\}
$$

Then, in the lattice framework, we define two operators $e(M)$ and $d(M)$ on the complete lattice $\mathcal{L}$ by :

$$
\begin{array}{r}
\forall M \in \mathcal{M}(S), \\
e(M)=\left\{T \in \operatorname{Del}, e_{T}\right\} \text { and } d(M)=\left\{T \in \operatorname{Del}, d_{T}\right\}
\end{array}
$$

with $e_{T}$ and $d_{T}$ defined in Eq. 2.

We proved in [3] that $e(M)$ and $d(M)$ are respectively erosion and dilation morphological operators on $\mathcal{L}$. We can define various operators now like opening $o(M)$ end closing $c(M)$ :

$$
\begin{array}{r}
\forall M \in \mathcal{M}(S)), \\
o(M)=d \circ e(M) \text { and } c(M)=e \circ d(M)
\end{array}
$$

and benefits of all the inherited operators based on the erosion and the involution operators ${ }^{c}$ :

$$
\forall M \in \mathcal{M}(\operatorname{Del}(S)), M^{c}=\left\{T \in \operatorname{Del}, 1-\phi_{T}\right\}
$$

Thus, as expected,

$$
\forall M \in \mathcal{M}(\operatorname{Del}(S)), e(M)=d\left(M^{c}\right)^{c}
$$

\section{Modeling spatial relations}

1) The directional dilation: The whole set of spatial relation concepts modeling is based on the directional dilation operator. Let $\alpha$ be the directional angle with regard to the horizontal axis of the representation plane. For any mesh $M \in \mathcal{L}$, the definition of the neighborhood $\nu(T)$ of a mesh triangle $T$ acts as a directional structural element of direction $\alpha$ :

$$
\nu_{\alpha}(T)=\left\{T^{\prime} \in \operatorname{Del} \mid T^{\prime} \cap T \neq \emptyset \text { and } \angle\left(T, T^{\prime}\right)<\alpha+\epsilon\right\}
$$

with

$$
\angle\left(T, T^{\prime}\right)=\angle\left(\overrightarrow{B_{T} B_{T^{\prime}}},(O \vec{x})\right)
$$

where $B_{T}$ is the barycenter of the triangle $T$.

Eq. 8 defines an anisotropic neighborhood referred as $\nu_{\alpha}$, to be put in parallel with the isotropic neighborhood definition $\nu_{\text {iso }}$ of equation 3, where $\alpha$ stand for the angle with the horizontal axis $(O \vec{x})$ and $\epsilon$ is the width or tolerance angle of the structuring element.

We can define the directional dilation as:

$$
\forall M \in \mathcal{M}(S), d_{\alpha, \epsilon}(M)=d_{\nu_{\alpha}}(M)
$$

where $d_{\text {neighborood }}$ stands for the computation of $d_{T}$ values over a specific system of neighborhood. When $\epsilon$ is not written, $\epsilon=\pi / 8.0$ and $d_{\alpha, \epsilon}=d_{\alpha}$.

Qualitative concepts like spatial relations depend both on the context and on the reference object. A fuzzy version of for all the morphological operators acting on meshes following the algorithmic procedure in Alg. 1 can deliver more flexible results for reasoning purposes. From this fundamental spatial relation, by composition, we can define a bunch of qualitative spatial relation in a quantitative way such as "between" as briefly illustrated in the following section.

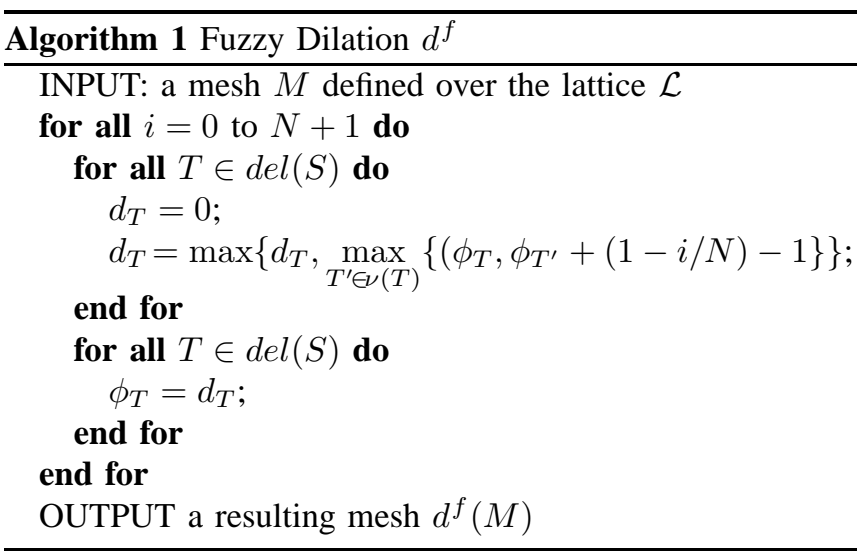

2) The relation "South East": Directional relations can now be defined in a crisp or a fuzzy mode over any point set. For instance, the absolute directional spatial relation like "south east of" is defined as follows:

$$
\begin{aligned}
& \text { SouthEast }_{d i l}\left(M_{1}\right)=d_{7 \pi / 4}^{n}\left(M_{1}\right) \\
& \text { with } n / d_{7 \pi / 4}^{n}\left(M_{1}\right)=d_{7 \pi / 4}^{n+1}\left(M_{1}\right)
\end{aligned}
$$

where $d^{n}$ stands for $d \circ d \circ \ldots \circ d n$ times. The SouthEast ${ }^{f}$ fuzzy operator is directly obtained by combining the definitions 10 and 11 associated with the directional neighborhood $\nu_{\alpha}$ into the algorithmic procedure used to compute $d^{f}$. In the following Sec. III, we extent both the scope of spatial relations and of use cases for microscopic as well for macroscopic images.

\section{RESULTS AND APPLICATIONS}

In this section, we illustrate the efficiency of these tools either to perform automatic analysis or to interact in a semiautomatic way with the visual data in the perspective of a visual cognition paradigm. We achieved structural analysis results over a variety of images (macroscopic/microscopic, natural/document) to assess the effectiveness of the proposed toolbox.

\section{A. Structural analysis}

The Lukas-Kanade tracking algorithm of objects over video sequence is based on multi-scale robust point set extractors. We illustrate the structural analysis that can be performed onto this specific sparse representation of a macroscopic image in Fig. 3 and Fig. 4. For the extraction 
of the starting sub-mesh of interest, we use the concept of $\alpha$-objects [9], [8], [3]. The mapping $\phi$ associates to each triangle $T$ a $\phi_{T}$ value corresponding to intrinsic properties of the triangles. For Fig. 3(b), $\phi_{T}$ corresponds to a measure of the size of the triangle $T$ defined by its three vertices A,B,C: $\phi_{T}=\max (A B, A C, B C)$. For Fig. 4(a), $\phi_{T}$ corresponds to a measure of the shape of the triangle: $\phi_{T}=1 / \rho_{T}$, with $\rho_{T}$ the radius of the circumscribed sphere to $T$. The starting sub-mesh corresponds to the simplicial complex $C(S)=T \in \operatorname{Del}(S), \phi_{T}$. Then, the $\theta$ threshold is chosen as a median value: $\theta_{\text {opt }}=0.5 *$ median $_{T \in D e l}\left(\phi_{T}\right)$. The starting sub-mesh of interest is $M=\operatorname{bin}_{\theta}(C(S)$. Then, various morphological operators based on the basic erosion (and dilation) operator can derive relevant structural outcomes, in particular outlining face features such as the eyes and the mouth regions (see Fig. 3(d) and (f)). Note that the operators work as well on triangles as on edges in a contour mode [3]. The mouth region and eyes region are obtained in Fig. 3(f) as open-like mesh filtered:

$$
\text { MouthAndEyes }(M)=d \circ e \circ e(C)
$$

Fig. 4 illustrates an another result if we choose the shape criterion for $\phi_{T}$.

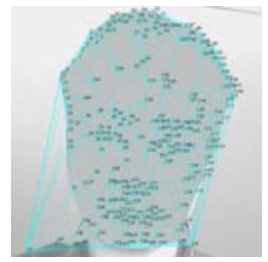

(a)

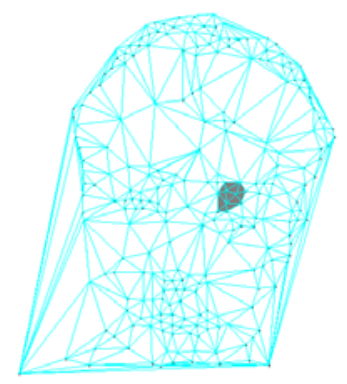

(b)
Fig. 4. (a) Thresholding with the Shape Criteria $M_{\text {shape }}$ (b) Order 5 opening-like filtering of $M_{\text {shape }}: d \circ e^{5}(C(S)$

\section{B. Document analysis}

In [14], the logical structure of a document image with irregular layout - like the location of title and author regions - is determined by the way of a Delaunay triangulation-based method. This method is very robust and can also establish the major threshold values, such as the line space and the inter-character space, automatically, from a document image itself. Some features are measured from different classified Delaunay triangle groups in the Delaunay triangulation model. These feature measures can be improved by the use of morphological mesh operators to clean out the shape of the various regions in the document.

Based on the mass centers of the connected components, Fig. 5 presents such ideas. The gray area corresponds to the dilated mesh for an $\theta$ threshold of $\theta_{\text {opt }}=0.75 *$ median $_{T \in D e l}\left(\phi_{T}^{\text {size }}\right)$ and extracts the specific text area in the document not related to the title areas.

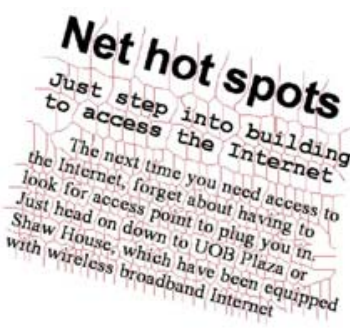

(a)

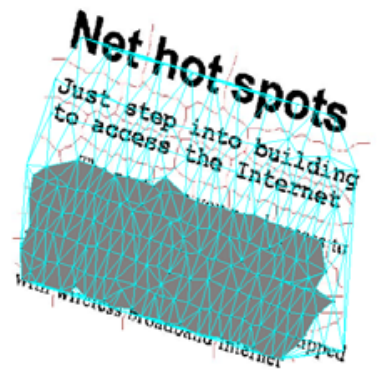

(b)
Fig. 5. (a)Mass centers of the connected components, (b) the gray area corresponds to the dilated of the mesh $\operatorname{bin}_{\theta}(C(S))$

\section{High-level spatial relation queries}

In histopathological images, the need to explore highcontent, large images to draw a diagnosis makes it relevant the development of smart interfaces to perform high-level semantic queries involving spatial relations [12]. The diagnosis of breast cancer for instance is based on the analysis of spatial distributions of cells and lumina, which are the mammal ducts to be short. The answer to the query "Show me the cells around the lumina" or the action "Digitalize the cell images between the lumina1 and the lumina2" in the case of a motorized microscope are of major importance to enhance the diagnosis process and to make it possible the digitalized exploration of such data. As for now, the $\phi_{T}=1$ values will be displayed white triangles and the $\phi_{T}=0$ values will be displayed as transparent triangles. Fig. 6 illustrates two such biological lumina under the form of two meshes $M_{1}$ and $M_{2}$ (Fig. 6 (b)).

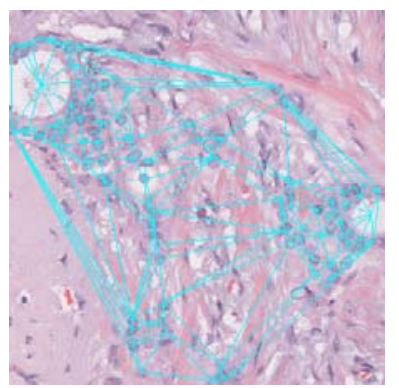

(a)

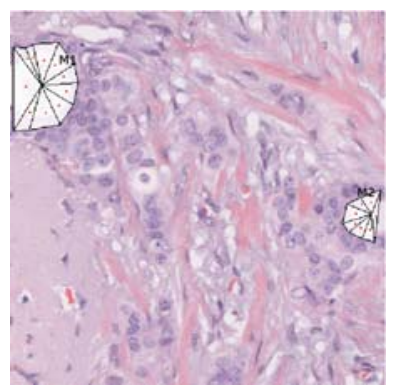

(b)
Fig. 6. (a) A small 1024x1024 pixel size images out of a Whole Slide Image (b) Two sub-meshes of interest $M_{1}$ and $M_{2}$ corresponding to two biological lumina objects.

Fig. 7 illustrates the successive directional dilations of the relation SouthEast $\left(M_{1}\right)$ until stabilization yielding to the south-east crisp region of the lumina $M_{1}$. In Fig. 7(h) a filtered version by an final isotropic opening is provided: $o_{\nu_{\text {iso }}}\left(d_{7 \pi / 4}^{n}\left(M_{1}\right)\right)$.

Fig. 8 illustrates the between relation between $M_{1}$ and $M_{2}$ in a crisp definition (Fig. 8(e)) and in a fuzzy definition (Fig. 8(f)). Let $\alpha$ be the angle between the $x$ axis and the line joining the centers of gravity of $M_{1}$ and $M_{2}$. Fig. 8(a) and (b) illustrate the $d_{\alpha}^{n}\left(M_{1}\right)$ and $d_{2 \pi-\alpha}^{n}\left(M_{2}\right)$ sub-meshed 
a theoretical toolbox to enhance the cognitive experience with handling visual data and leverage interactive queries to improve practical application outcomes.

\section{ACKNOWLEDGMENT}

The authors acknowledge the support of Ludovic Roux - IPAL for the cognitive virtual microscope project and Jacques Klossa - TRIBVN and National University Hospital of Singapore for providing histopathological images and inspiring fruitful discussions about the new challenges in microscopic imaging systems.

\section{REFERENCES}

[1] E. Clementini, P. Di Felice, Approximate Topological Relations, International Journal of Approximate Reasoning (16) (1997) 173-204.

[2] P. Matsakis, L. Wendling, A new way to represent the relative position between real objects, IEEE Transactions on Pattern Analysis and Machine Intelligence 21 (7) (1999) 634-643.

[3] N. Lomenie, G. Stamon, Morphological Mesh filtering and alphaobjects, Pattern Recognition Letters 29 (10) (2008), 1571-1579.

[4] O. Colliot, 0. Camara, I. Bloch, Integration of Fuzzy Spatial Relations in Deformable Models - Application to Brain MRI Segmentation, Pattern Recognition (8) (2006) 1401-1414.

[5] V.T. Ta, O. Lezoray, A. Elmoataz, S. Schupp, Graph-based Tools for Microscopic Cellular Image Segmentation, Pattern Recognition, Special Issue on Digital Image Processing and Pattern Recognition Techniques for the Detection of Cancer 42 (6) (2009) 1113-1125.

[6] ECVision: The European Research Network for Cognitive Computer Vision Systems, "A Research Roadmap of Cognitive Vision", IST Project IST-2001-35454, August 2005.

[7] I. Bloch, O. Colliot, R.M. Cesar, On the ternary spatial relation "between", IEEE Trans. on Systems, Man, and Cybernetics, Part B: Cybernetics 36 (2) (2006) 312-327.

[8] H. Edelsbrunner, D.G. Kirkpatrick, On the shape of set of points in the plane, IEEE Trans. on Information Theory (29) (1983) 551-559.

[9] H. Edelsbrunner, E.P. Mucke, E.P., Three-dimensionnal alpha-shapes, ACM Transactions on Graphics 13 (1) (1994) 43-72.

[10] H. Heijmans, P. Nacken, A. Toet, L. Vincent, Graph Morphology, Journal of Visual Communication and Image Representation 3 (1) (1992) 24-38.

[11] L. Vincent, Graphs and Mathematical Morphology, Signal Processing 16 (4) (1989) 365-388.

[12] S. Doyle, S. Agner, A. Madabhushi, M. Feldman, J. Tomaszewski, Automated Grading of Breast Cancer Histopathology Using Spectral Clustering with Textural and Architectural Image Features, in: 5th IEEE International Symposium on Biomedical Imaging: From Nano to Macro, ISBI, (29) (2008) 496-499.

[13] J. Cousty, L. Najman, J. Serra, Some morphological operators in graph spaces, in: Computer Sciences Springer, Lecture Notes (ed), Mathematical Morphology and Its Application to Signal and Image Processing, vol. 5720 (2009) 149-160.

[14] Y. Xiao, H. Yan, Location of title and author regions in document images based on the Delaunay triangulation, Image and Vision Computing, vol. 22 (2004) 319-329. 